\title{
A CT-scan compatible robotic device for needle placement in medical applications
}

\author{
Calin Vaida ${ }^{1, a}$, Bogdan Gherman $^{1, b}$ Doina Pisla ${ }^{1, c}$ and Nicolae Plitea ${ }^{1, d}$ \\ ${ }^{1}$ Technical University of Cluj-Napoca, Faculty of Machine Building, Department of Mechanical \\ Systems Engineering, Romania \\ acalin.vaida@mep.utcluj.ro, bbogdan.gherman@mail.utcluj.ro (corresponding author), \\ cdoina.pisla@mep.utcluj.ro, 'nicolae.plitea@mep.utcluj.ro
} Keywords: kinematic modeling, spherical mechanism, CT-scan compatible device, needle
placement

\begin{abstract}
Several medical applications require devices capable of placing different substances inside the human body. Due to the nature of the task it is desirable to perform these actions with visual feedback, whereas the most viable solution, especially for deep target points, is computer tomography (CT). The paper presents an innovative device, which can be fitted inside the CT gantry, and has decoupled motions to ensure maximum accuracy during the needle placement. It will be shown that for needle placement tasks 5 degrees of freedom (DOF) are sufficient to achieve the task. The geometric and kinematic model of the robot will be presented. The workspace and precision mapping are computed. Some simulation results will show the robot capabilities as well as its placement in the CT scan environment.
\end{abstract}

\section{Introduction}

"A wise man should consider that health is the greatest of human blessings, and learn how by his own thought to derive benefit from his illnesses." (Hippocrates)

Needle placement technique covers a wide area of medical applications, where the task can be described, in a very simple way, as the insertion of a needle on a linear trajectory, from outside up to a target point situated in the patient body. Needle placement covers applications like biopsies, fluid extraction from internal organs (cardiac tamponade, lungs) or placement of radioactive seeds in different body organs in minimally invasive cancer therapy [1-3]. In [1] it is shown that a robotic device enhances the needle placement precision beyond the natural human capabilities. Most robotic devices developed for needle placement applications are dedicated for a single, very specific task, thus narrowing its range of applications to a singular one.

Application definition. The robotic structure should introduce, based on radiologic data, rigid needles of diameter varying from $0.6 \mathrm{~mm}$ up to $2 \mathrm{~mm}$ and a length from $50 \mathrm{~mm}$ up to $250 \mathrm{~mm}$ inside the patient body following a linear trajectory. Due to the long distances, the variable tissue density one can define the first requirement for the robot: the needle is introduced, in a linear trajectory by an independent mechanism, to ensure constant precision for the entire displacement and to avoid any deviations from the predefined trajectory.

The areas of interest for needle placement in different medical applications where the use of a robotic device is justified cover the human thoracic and abdominal area where the target point can be situated in close vicinity to different structures (blood vessels, ganglions, other organs) whereas an error can cause a cataclysmic event leading up the death of the patient. Several applications require the placement of multiple needles in a circular or rectangular matrix display thus defining the second requirement for the robot: it should be capable of placing multiple needles on parallel trajectories. Thus an advantage would be to be able to fix the robot orientation after its initial setup and modify only the insertion point from one needle to the other.

Besides emergency situations where the intervention must be performed very fast, needle placement is carried out following a set of imagistic investigations ranging from simple radiologic exams to echography, computer tomography (CT) and magnetic resonance imaging (MRI). These techniques provide a set of images enabling the preplanning of the procedure where the physician 
defines safe linear trajectories to reach the targeted point(s). Nevertheless, in order to increase the safety of the procedure it is desired to have a real-time visual feedback of the needle placement during the procedure to reduce the risk of damaging any internal body structures [2]. During the first steps of introducing robotic systems in medical arena, the imagistic methods that can be used during the needle placement were defined [3,4]: High Intensity Focal Ultrasound (HIFU), CT, MRI and Positron Emitted Tomography (PET Scan). Each of these techniques has advantages and limitations. The best option as image quality is the use of MRI but due to the high magnetic fields generated by the machine there are a lot of restrictions for both patient and robot. The ultrasound machines have a low image quality and a limited use especially for deep targets. CT can be considered at the moment as the most versatile option even though the image quality is lower compared to MRI machines but it is still good enough for real-time needle placement procedures. Figure 1 illustrates an example of a CT machine equipped with supplementary laser sensors (the lasers were enhanced in the figure to provide better contrast) for position calibration. This type of CT scanners is designed especially for fast real-time image feedback but they cannot be used in diagnostics [5]. Figure 2 shows a CT image with a needle inserted inside the human body [6].

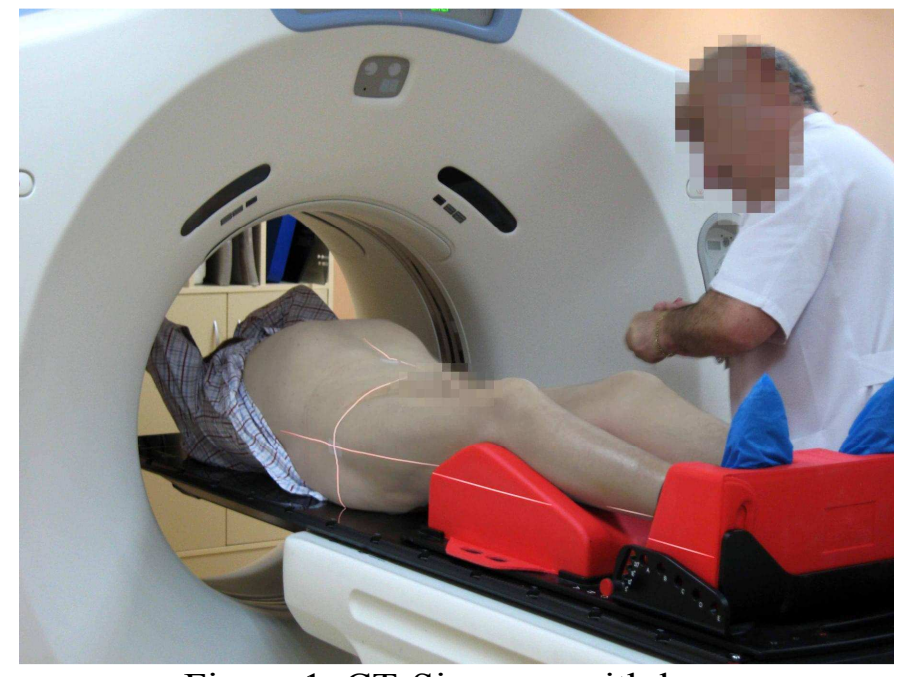

Figure 1. CT-Sim scan with laser positioning system

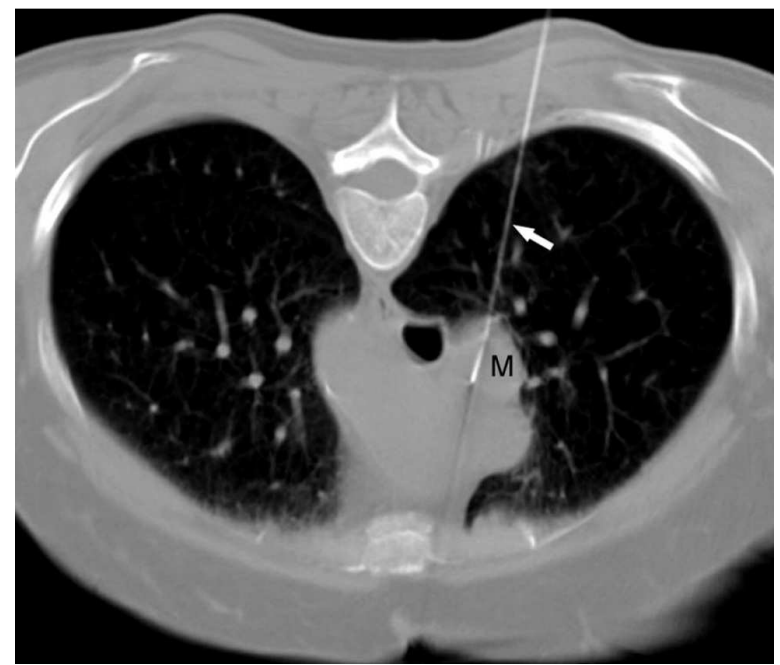

Figure 2. CT scan with needle placement (arrow) for transpulmonary biopsy [6]

The need to provide real-time visual feedback for the needle position during its insertion defines the next technical characteristic of the robotic system: it must be small enough to fit inside the CT gantry in the patient proximity.

\section{Definition of an innovative robotic structure}

In [1] the needle placement precision of manual template guidance was calculated to be around 3 $\mathrm{mm}(2.7 \pm 0.7 \mathrm{~mm})$. Many applications require a higher precision $(1 \mathrm{~mm})$ making the manual task impossible to achieve. Considering the fact that the target point inside the body is known and the trajectory is defined, it means that also the entrance point is known, and being visible it is very easily and precisely reached. This means that the error is generated mainly by the orientation angle of the needle. A simple estimation of the acceptable angular deviation for a maximum error of 1 $\mathrm{mm}$ is given by Eq. 1 where $\mathrm{d}$ represents the trajectory length inside the body and $\alpha_{\max }$ is the angular deviation:

$$
\alpha_{\max }=2 \cdot \operatorname{asin}\left(\frac{1}{2} \cdot \frac{1}{\mathrm{~d}}\right) \cdot \frac{180}{\pi}
$$


Taking for example a depth of $250 \mathrm{~mm}$, the maximum angular deviation is:

$$
\alpha_{\max }=2 \cdot \operatorname{asin}\left(\frac{1}{2} \cdot \frac{1}{250}\right) \cdot \frac{180}{\pi}=0.2292\left[^{\circ}\right]
$$

a value which is really small, taking into account also the tissue deformability.

Referring to the trajectory definition, in needle placement applications, one can generate the displacement as the line which connects the two control points: the target point and the insertion point. Analyzing the two points only the target point is totally fixed and the correct position of the robot can be verified only after the needle insertion. Thus, it is very hard to ensure that the trajectory is achieved, introducing some limitations in the overall robot precision and during the procedure very often the needle must be retracted and inserted again.

In order to improve the needle insertion technique an innovative concept is introduced which enables the definition of a supplementary control point, with fixed coordinates which together with the target point generated a line in space. This line provides the data for the needle orientation in space and the robot trajectory can be easily corrected before the actual insertion if the structure can ensure that the needle will always pass through the additional control point defined. The additional control point which is fixed in space is known as remote center of motion (RCM) and this concept was used generally in minimally invasive procedures where the RCM represented the insertion point in the patient body. The concept of RCM was introduced by Taylor [7] in the early ages of robotic surgery, and it can be achieved in three ways: using the natural joint generated by the patient body (by adding a 2-DOF passive joint at the coupling point of the robot and the surgical instrument [8]) mechanically constrained (by using 2 additional active joints which orient the mechanism around the insertion point [9]) and architecturally constraint (when the structure is designed in such a way that it will always pass through a fixed point in space). In [10] Beira et al present a synthesis of passive and mechanically constrained solutions for obtaining the remote center of motion (RCM) and propose an innovative parallel manipulator with 4 DOF and a fixed RCM. Arata et al develop in [11] a neurosurgical robotic system for brain tumor removal with a fixed RCM consisting of a 4 DOF active mechanism and a 3 DOF passive one. Marcu presents in [12] a set of algorithms for a motion simulator useful for the structure assessment before a prototype is built.

For the needle placement applications the second control point can be very useful if it is obtained as an architecturally fixed RCM. Due to the nature of the trajectory inside the human body, a linear displacement with a given orientation, the RCM can be situated on any point of the trajectory. In [13] Vaida et al present a concept which combines the simplicity of the structures that use a natural RCM with the positioning capabilities of the ones that have a mechanically constraint RCM by developing a pure spherical mechanism which provides an architecturally fixed RCM in the center of the sphere. Advanced design techniques for shape and workspace optimization are presented in [14] while in [15] the authors present solutions for the robot control improvement.

Fig. 3 illustrates the kinematic scheme of a spherical robotic arm with three active joints: The first motor achieves a rotation around the $\mathrm{X}$ axis, the second one achieves a translation along a curve of radius $\mathrm{R}$ while the third motor achieves a linear displacement along the radius of the sphere. The center of the sphere becomes the second control point through which the needle will always pass. From the kinematic point of view the only functional parameter which determines the robot size is the radius $\mathrm{R}$. From the spherical mechanism point of view, the center of the sphere is fixed, thus when the orientation is achieved the needle will be situated on a parallel line with the defined needle trajectory. This means that this line must be superposed over the real trajectory and this can be achieved with an XY positioning system on which the spherical mechanism is positioned on. Thus, in Fig. 4 is presented the kinematic scheme of the innovative 5-DOF robotic arm, called ATEN, optimized for needle placement interventions, consisting in a 2-DOF planar mechanism with intersected axis which achieves the displacement with constant orientation of the mobile platform in a plane with $Z=0$. A fixed coordinate system $O X Y Z$ is attached to the robot with the origin in the middle of the platform where the coordinates of the planar mechanism are $\left(\mathrm{q}_{4}=0\right.$ and $\mathrm{q}_{5}=0$ ). On top of the mobile platform it is positioned the 3-DOF spherical mechanism which has 


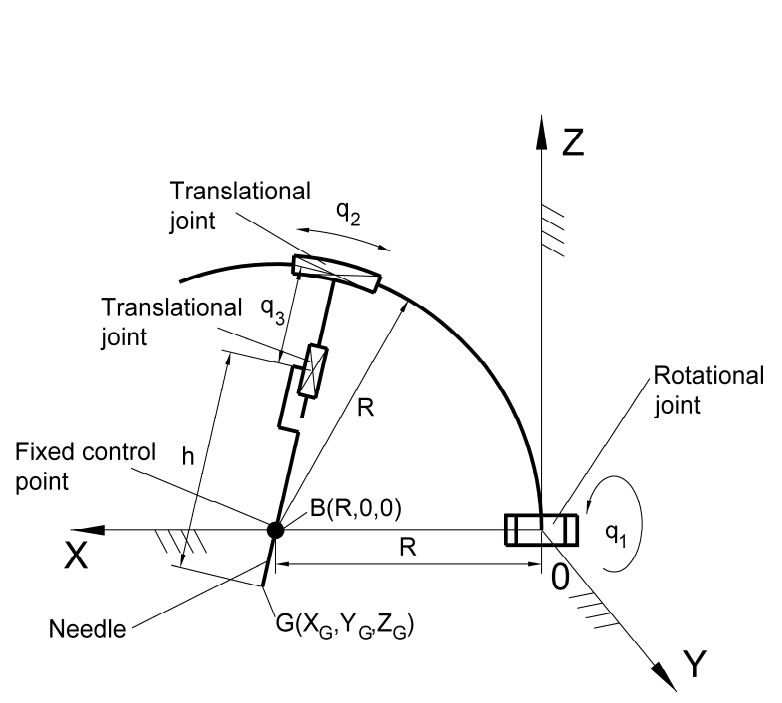

Fig. 3 The kinematic scheme of the spherical structure

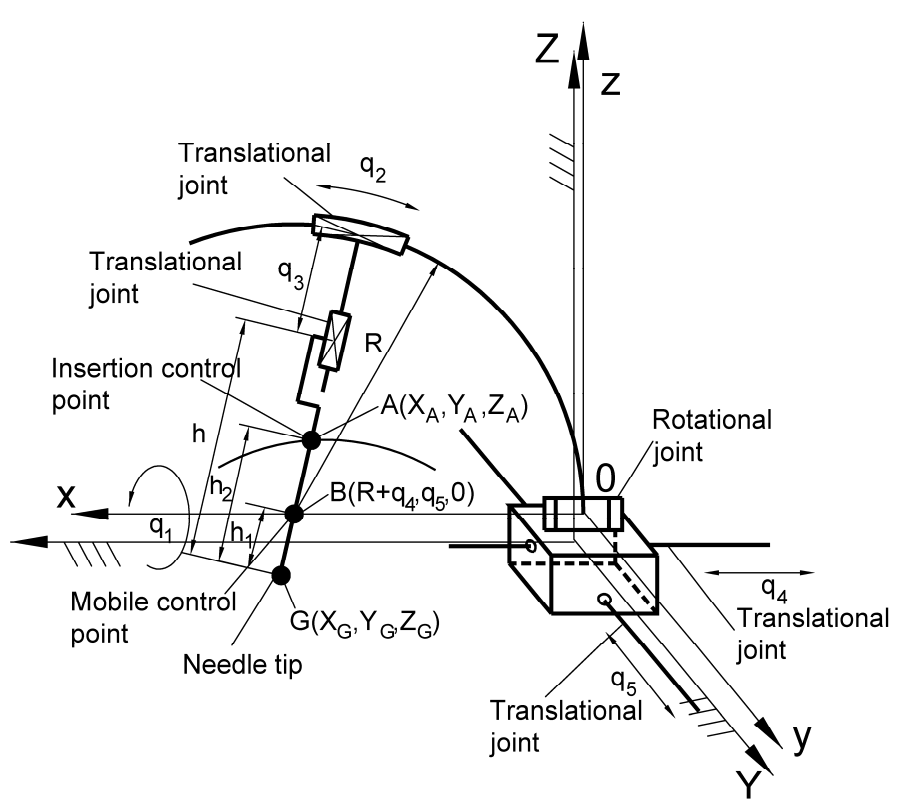

Fig. 4 The kinematic scheme of the 5-DOF robotic arm ATEN

a mobile coordinate system OXYZ with the origin in the center of the rotational joint and the axes parallel with the ones of the fixed coordinate system.

\section{Geometric modeling}

For the geometric modeling of the 5-DOF robotic arm the following parameters are introduced:

- $\mathrm{R}$ - the radius of the sphere;

- $\mathrm{h}$ - the length of the needle;

- $\mathrm{B}(\mathrm{R}, 0,0)$ - the coordinates of point $\mathrm{B}$ with respect to the mobile coordinate system, OXYZ;

- $\mathrm{B}\left(\mathrm{R}+\mathrm{q}_{4}, \mathrm{q}_{5}, 0\right)$ - the coordinates of point $\mathrm{B}$ with respect to the fixed frame, OXYZ.

For the mathematical simplicity of the model one and based on the fact that the fixed and mobile coordinate systems have the origin in the same plane $(\mathrm{Z}=\mathrm{Z}=0)$ and the axes are parallel the motion of the ATEN robotic arm can be studied individually for the spherical module and the XY positioning table. For computing the geometrical model, the following matrices are introduced:

Rotation around the $\mathrm{x}$ axis of the mobile system with $q_{1}$ :

$$
R x_{-} q_{1}=\left[\begin{array}{ccc}
1 & 0 & 0 \\
0 & \cos \left(q_{1}\right) & -\sin \left(q_{1}\right) \\
0 & \sin \left(q_{1}\right) & \cos \left(q_{1}\right)
\end{array}\right]
$$

Rotation around the y axis of the mobile system with $q_{2}$ :

$$
R y_{-} q_{2}=\left[\begin{array}{ccc}
\cos \left(q_{2}\right) & 0 & \sin \left(q_{2}\right) \\
0 & 1 & 0 \\
-\sin \left(q_{2}\right) & 0 & \cos \left(q_{2}\right)
\end{array}\right]
$$


Translation on the $\mathrm{z}$ axis of the mobile system with $-q_{3}-h$ :

$$
T z_{-}-q_{3}-h=\left[\begin{array}{c}
0 \\
0 \\
-q_{3}-h
\end{array}\right] \text {. }
$$

For the XY positioning table, two translations are defined: the first one with $q_{4}$ along the $\mathrm{X}$ axis and the second one with $q_{5}$ along the $\mathrm{Y}$ axis, in both cases measured with respect to the fixed coordinate system:

$$
\begin{aligned}
& T_{-} X_{-} q_{4}=\left[\begin{array}{c}
q_{4} \\
0 \\
0
\end{array}\right] . \\
& T_{-} Y_{-} q_{5}=\left[\begin{array}{c}
0 \\
q_{5} \\
0
\end{array}\right] .
\end{aligned}
$$

The authors adopted the XY Euler convention for the rotational matrices which lead to the following data. Rotation around the $\mathrm{x}$ axis with $\psi$ :

$$
R x_{-} \psi=\left[\begin{array}{ccc}
1 & 0 & 0 \\
0 & \cos (\psi) & -\sin (\psi) \\
0 & \sin (\psi) & \cos (\psi)
\end{array}\right] .
$$

Rotation on the y axis with $\theta$ :

$$
R y_{-} \theta=\left[\begin{array}{ccc}
\cos (\theta) & 0 & \sin (\theta) \\
0 & 1 & 0 \\
-\sin (\theta) & 0 & \cos (\theta)
\end{array}\right] \text {. }
$$

Where the two angles, $\psi$ and $\theta$ have the expressions:

$$
\begin{aligned}
& \psi=\operatorname{atan} 2\left(y_{G}-y_{B}, z_{B}-z_{G}\right) . \\
& \theta=\operatorname{atan} 2\left(x_{B}-x_{G}, \frac{\left(z_{B}-z_{G}\right)}{\cos (\psi)}\right) .
\end{aligned}
$$

Due to the fact that the mobile and fixed coordinate systems have parallel axes, the value of the $\psi$ and $\theta$ angles doesn't change from one system to the other.

\section{The inverse geometrical model}

For the inverse geometrical model the geometrical parameters of the structure and the coordinates of the needle tip (point $G$ ) are considered known and the coordinates of the active joints $q_{1}, \ldots, q_{5}$ should be determined. Furthermore, the insertion point in the body, denoted with $\mathrm{A}$ is given in absolute coordinates. 
From the kinematic scheme in figure 4, one can define the condition for the correct needle positioning (in the given control point G: the collinearity of the points G, B and A. This means that the angles $\psi$ and $\theta$ can be calculated with the following expressions:

$$
\begin{aligned}
& \psi=\operatorname{atan} 2\left(Y_{G}-Y_{A}, Z_{A}-Z_{G}\right) . \\
& \theta=\operatorname{atan} 2\left(X_{A}-X_{G}, \frac{\left(Z_{A}-Z_{G}\right)}{\cos (\psi)}\right) .
\end{aligned}
$$

Using equations (1) - (13) it results:

$\left\{\begin{array}{l}q_{1}=\operatorname{atan} 2(\sin (\psi) \cdot \cos (\theta), \cos (\psi) \cdot \cos (\theta)) \\ q_{2}=\operatorname{atan} 2\left(\sin (\theta) \cdot \cos \left(q_{1}\right), \cos (\psi) \cdot \cos (\theta)\right) . \\ q_{3}=R-\left(h-h_{1}\right)\end{array}\right.$

Where:

$$
h_{1}=\left|\frac{Z_{B}-Z_{G}}{\cos (\psi)}\right|=\left|\frac{-Z_{G}}{\cos (\psi)}\right| \text {. }
$$

Knowing the distance between the points B and $\mathrm{G}\left(h_{1}\right)$ the $X_{B}$ and $Y_{B}$ coordinates can be computed, and thus, the values for the active joints $q_{4}$ and $q_{5}$ :

$$
\begin{aligned}
& \left\{\begin{array}{l}
X_{B}=X_{G}+h_{1} \cdot \sin \left(q_{2}\right) \\
Y_{B}=Y_{G}-h_{1} \cdot \sin \left(q_{1}\right) \cdot \cos \left(q_{2}\right)
\end{array}\right. \\
& \left\{\begin{array}{l}
q_{4}=X_{B}-R \\
q_{5}=Y_{B}
\end{array}\right.
\end{aligned}
$$

\section{The direct geometrical model}

For the direct geometrical model the geometrical parameters of the structure and the coordinates of the active joints $q_{1}, \ldots, q_{5}$ are considered known and the coordinates of the needle tip (point $\mathrm{G}$ ) should be determined. Furthermore, the insertion point in the body, denoted with $\mathrm{A}$ is given in absolute coordinates.

In the first step, the coordinates for the point B with respect to the fixed coordinate system are determined:

$$
\left\{\begin{array}{l}
X_{B}=R+q_{4} \\
Y_{B}=q_{5} \\
Z_{B}=0
\end{array}\right.
$$

The expressions of the angles $\psi$ and $\theta$ are determined using the known values of $q_{1}$ and $q_{2}$ :

$$
\left\{\begin{array}{l}
\psi=\operatorname{atan} 2\left(\sin \left(q_{1}\right), \cos \left(q_{1}\right)\right) . \\
\theta=\operatorname{atan} 2\left(\sin \left(q_{2}\right), \cos \left(q_{2}\right)\right)
\end{array}\right.
$$


The length of the needle below the sphere center is:

$$
h_{1}=q_{3}-R+h \text {. }
$$

Using the known coordinates of point $\mathrm{B}$, it results:

$$
\left\{\begin{array}{l}
X_{G}=X_{B}-h_{1} \cdot \sin \left(q_{2}\right) \\
Y_{G}=Y_{B}-h_{1} \cdot \sin \left(q_{1}\right) \cdot \cos \left(q_{2}\right) \\
Z_{G}=-h_{1} \cdot \cos \left(q_{1}\right) \cdot \cos \left(q_{2}\right)
\end{array}\right.
$$

\section{Workspace and singularity analysis of the 5-DOF robotic system, ATEN}

As it was shown in the first paragraph, the application definition, a critical condition for the robotic system ATEN is to fit inside the gantry of the CT scan in order to enable real time visual positioning control for the needles during their insertion. For that the dimensions of the robot must be correlated with the CT machine. Figure 1 illustrates a typical CT-Sim device, with the following parameters: Gantry opening diameter: $800 \mathrm{~mm}$; $\bullet$ Vertical laser positioning: $110 \mathrm{~mm}$ in front of the gantry (see figure 1); $\downarrow$ Lateral, horizontal lasers, in the plane passing through the gantry center; - The mobile couch $110 \mathrm{~mm}$ below the median plane of the gantry.

A simple way to use the space inside the gantry very efficiently is to keep the rotation axis of the first active joint, $q_{1}$, in the plane defined by the horizontal laser. In this situation, the radius of the spherical mechanism will be concentric with the gantry, ensuring the free motion of the mechanism without any risks of damaging the gantry. For the radius of the mechanism a value of $300 \mathrm{~mm}$ is considered to provide enough room around the patient. The workspace of the robot will be computed for a needle with a total length of $200 \mathrm{~mm}$. Figure 5 illustrates the robot workspace for a fixed position of the point $\mathrm{B}$ (without any actuation on $q_{4}$ and $q_{5}$ ). In figure 5, analyzing the robot workspace it can be easily seen that it covers properly the entire half of the patient situated in the robot proximity, meaning that the robot has to be mounted proximal to the patient side where the tumor is located. Figure 6 represents the total workspace of the robot with a displacement of 100 mm for $q_{4}$ and $200 \mathrm{~mm}$ for $q_{5}$, values selected accordingly to the space inside the CT-Sim gantry.

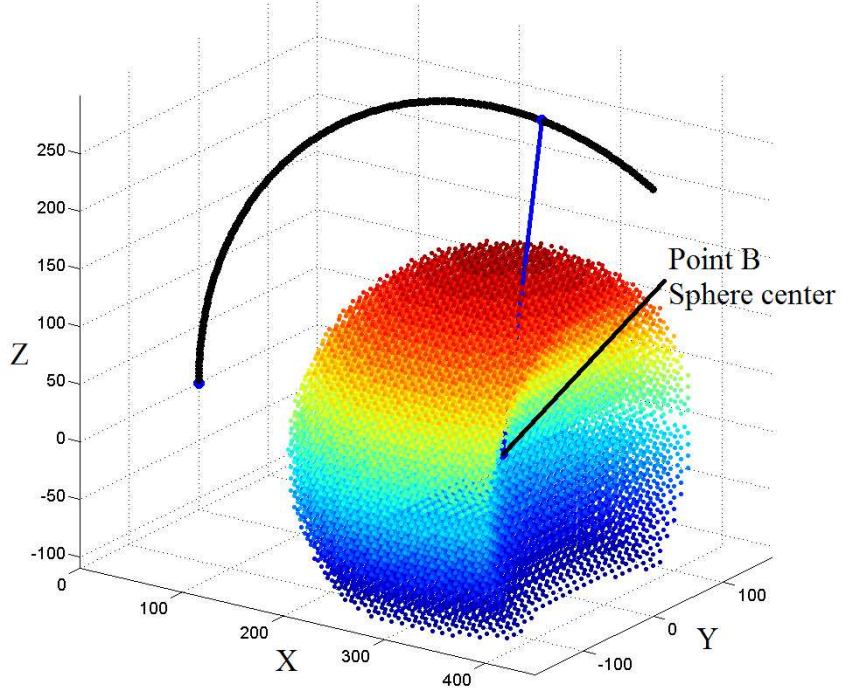

Figure 5. Robot workspace for a fixed location of the point $B$

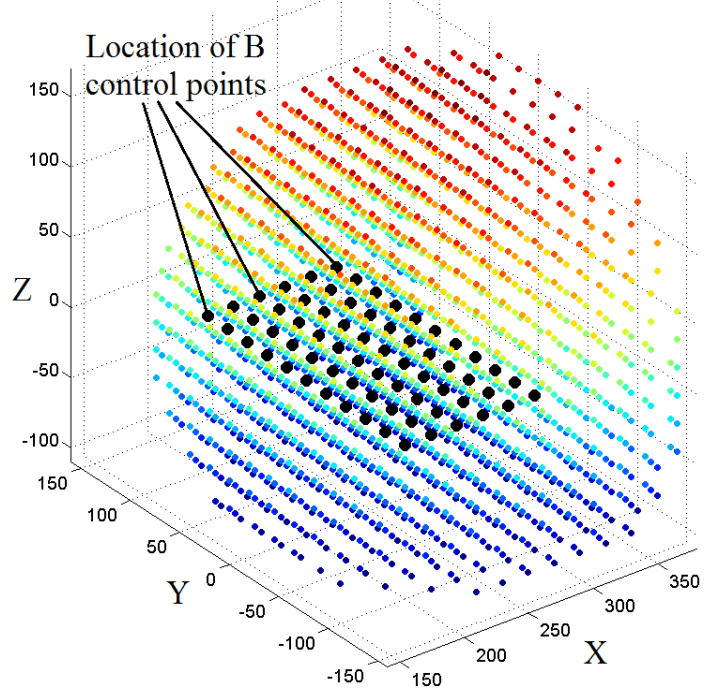

Figure 6. Total robot workspace

Singularities analysis. For the singularities analysis, the Jacobi matrices A and B must be computed. In order to reduce the computational time, as the axes $q_{4}$ and $q_{5}$ modify only the position of the sphere center, the singularities analysis can be performed for a fixed value of these 
two active joints, which will be treated as constants in the matrices. Thus, the Jacobi matrices will have $3 \times 3$ dimensions. The implicit equations which determine the relation between the active joints and the coordinates of the needle tip are:

$$
\left\{\begin{array}{l}
f_{1}\left(q_{1}, q_{5}, Y_{G}, Z_{G}\right) \equiv \frac{\sin \left(q_{1}\right)}{\cos \left(q_{1}\right)}+\frac{Y_{G}-q_{5}}{Z_{G}}=0 \\
f_{2}\left(q_{1}, q_{2}, q_{4} X_{G}, Z_{G}\right) \equiv \frac{\sin \left(q_{2}\right)}{\cos \left(q_{2}\right)}+\cos \left(q_{1}\right) \cdot \frac{R+q_{4}-X_{G}}{Z_{G}}=0 \\
f_{3}\left(q_{3}, q_{4}, q_{5}, X_{G}, Y_{G}, Z_{G}\right) \equiv q_{3}-R+h-h_{1}= \\
=q_{3}-R+h-\sqrt{\left(R+q_{4}-X_{G}\right)^{2}+\left(q_{5}-Y_{G}^{2}\right)+Z_{G}^{2}}=0
\end{array} .\right.
$$

Where: $h_{1}=\sqrt{\left(X_{B}-X_{G}\right)^{2}+\left(Y_{B}-Y_{G}\right)^{2}+\left(Z_{B}-Z_{G}\right)^{2}}$.

Using the Eq. 22, the Jacobi matrices are defined:

$$
\begin{aligned}
& A=\left(\begin{array}{ccc}
0 & \frac{1}{Z_{G}} & -\frac{Y_{G}-q_{5}}{Z_{G}^{2}} \\
-\frac{\cos \left(q_{1}\right)}{Z_{G}} & 0 & -\cos \left(q_{1}\right) \cdot \frac{R+q_{4}-X_{G}}{Z_{G}^{2}} \\
\frac{R+q_{4}-X_{G}}{h_{1}} & \frac{q_{5}-Y_{G}}{h_{1}} & \frac{Z_{G}}{h_{1}}
\end{array}\right) . \\
& B=\left(\begin{array}{ccc}
\frac{1}{\cos ^{2}\left(q_{1}\right)} & 0 & 0 \\
-\sin \left(q_{1}\right) \cdot \frac{R+q_{4}-X_{G}}{Z_{G}} & \frac{1}{\cos ^{2}\left(q_{2}\right)} & 0 \\
0 & 0 & 1
\end{array}\right)
\end{aligned}
$$

The determinant of matrix $\mathrm{A}$ is:

$$
\operatorname{det}(A)=\frac{\cos \left(q_{1}\right) \cdot h_{1}}{Z_{G}^{3}}
$$

Three cases are distinguished:

1. $q_{1}= \pm \frac{\pi}{2}$. This corresponds to the case when the spherical robot arm is placed horizontally on any of its sides, position that is improper for needle placement applications.

2. $h_{1}=0$. Geometrically, this means that $G \equiv B$. In this case, when the tip of the needle is exactly in the center of the sphere no matter how one modifies the values of $q_{1}$ and $q_{2}$, the position of the needle tip will remain the same. For needle placement applications, the needle is driven on a linear trajectory so this case has no effect on the robot maneuverability.

3. $Z_{G}=0$. This situation refers again to the boundary surface analyzed in the first case, and it results to be outside the operational robot workspace. 
The determinant of matrix $\mathrm{B}$ is:

$$
\operatorname{det}(B)=\frac{1}{\cos ^{2}\left(q_{1}\right) \cdot \cos ^{2}\left(q_{2}\right)}
$$

Two cases are distinguished:

1. $q_{1}= \pm \pi / 2$. This corresponds to the first case analyzed for the matrix A.

2. $q_{2}= \pm \pm \pi / 2$. The same situation is created when the second actuator reaches the extreme positions that places the tip of the instrument on the OXY plane at $Z_{G}=0$.

Analyzing the singularities presented above it can be concluded that there are no singular points in the operational workspace of the robot.

Needle insertion procedure definition. The ATEN robot is developed as a structure dedicated for needle placement applications, and in this paragraph the procedure description is presented as a sequence of logical and safety proof steps.

1. The medical data is received from the physicians defining the target points locations and the desired linear trajectories.

2. The patient is placed in the CT-Sim device and immobilized in a predefined position, then calibrated with respect the laser system of the machine.

3. The robot is rigidly fixed in the CT-Sim table and calibrated with respect to the laser system. After this step the robot must remain fixed until the procedure ends.

4. Base on the medical data provided, the needle is oriented parallel with the linear trajectory to be achieved. This is done using only the active joints $q_{1}$ and $q_{2}$.

5. The needle is superposed over the imposed trajectory, by means of the active joints $q_{4}$ and $q_{5}$.

6 . The needle is driven along the trajectory by means of the active joint $q_{3}$. When the needle touches the skin, due to the additional control point (the sphere center) the position is checked.

7. If needed, corrections are applied and then, the needle is inserted partially.

8. The needle position is checked again using the CT Sim device. If validated the needle is driven to the target point otherwise it is retracted and step 7 is repeated.

9. If multiple needles must be inserted, if the trajectories are pure parallel, steps 5-8 are repeated, otherwise steps 4-8 must be performed.

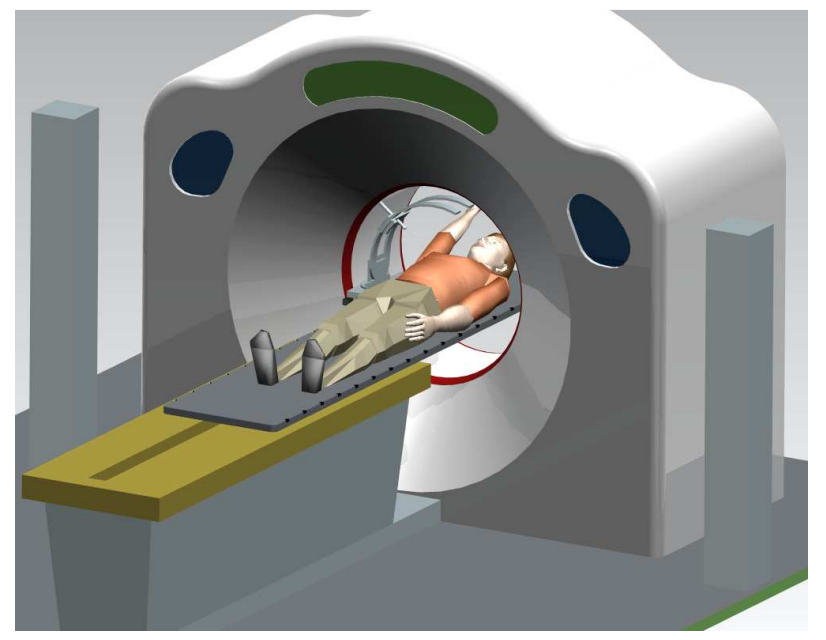

Figure 7. The ATEN robot in the CT-Sim environment

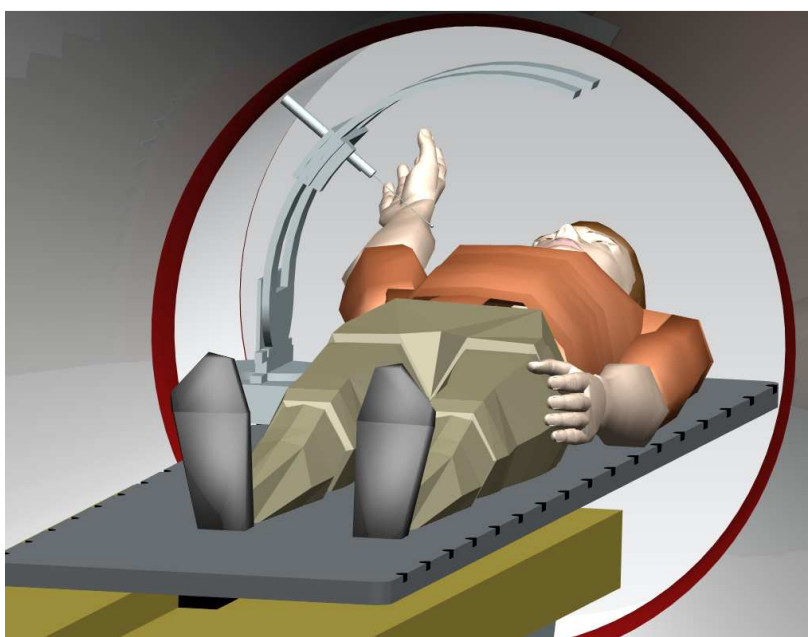

Figure 8. Detail showing the ATEN robot position inside the CT-Sim gantry

An illustration of the ATEN 5-DOR robotic arm placed in the CT-Sim device is presented in the figure 7 to show its dimensional relation to the machine and the patient as well as a detail showing its position inside the gantry, in figure 8 . 


\section{Summary}

The paper presents an innovative concept of a 5-DOF modular robot, design for needle placement techniques in medical applications. The design concept, based on the particularity of the spherical motion, is introduced and then motivated as a critical advantage for the given application. The geometrical modeling, workspace generation and singularities analysis are performed. The ATEN robotic system for needle placement is integrated as a component of the CT-Sim device, showing its capabilities to work under real-time visual feedback during the needle insertion procedure.

\section{Acknowledgment}

This paper was supported by a scholarship for the first author by the project "Development and support of multidisciplinary postdoctoral programmes in major technical areas of national strategy of Research - Development - Innovation" 4D-POSTDOC, contract no. POSDRU/89/1.5/S/52603, project co-funded by the European Social Fund through Sectoral Operational Programme Human Resources Development 2007-2013 and by logistics by the Scopes International Grant IZ74Z0137361 entitled Creative Alliance in Research and Education focused on Medical and Service Robotics (CARE-Robotics).

\section{References}

[1] G. Strassman et al, Advantage of robotic needle placement on a prostate model in HDR brachytherapy, Strahlenther Onkol. 187(6), (2011), 367-272.

[2] E. Boctor et al, Three-dimensional ultrasound-guided robotic needle placement: an experimental evaluation, Int J Med Robotics Comput Assist Surg, 4(2), (2008), 180-191.

[3] G.H, Ballantyne, Robotic surgery, telerobotic surgery, telepresence, and telementoring, Surgical Endoscopy, 16(10), (2002), 1389-1402.

[4] K. Cleary, C. Nguyen, State of the art in surgical robotics: Clinical applications and technology challenges, Computer Aided Surgery, 6(6), (2002), $312-328$

[5] D. Platten et al, GE LightSpeed RT CT scanner technical evaluation, Report 05070, National Health Service (NHS), 2005

[6] S. Gupta et al, Imaging-guided percutaneous biopsy of mediastinal lesions: different approaches and anatomic considerations, RadioGraphics, 25, (2005), 763-788

[7] R. H. Taylor, et al, A Telerobotic Assistant for Laparoscopic Surgery, IEEE Engineering in Medicine and Biology, (1995) 14, 279-287

[8] C. Vaida, et. al., Development of a Voice Controlled Surgical Robot, New Trends in Mechanism Science, Mechanisms and Machine Science, (2010) 5(10) 567-574

[9] D. Pisla, B. Gherman, C. Vaida and N. Plitea, Kinematic modelling of a 5-DOF hybrid parallel robot for laparoscopic surgery, Robotica, (2012), 30(07), 1095-1107

[10] R. Beira, et al, Dionis: A novel remote-center-of-motion parallel manipulator for Minimally Invasive Surgery, Applied Bionics and Biomechanics, (2011) 8(2) 191-208

[11] J. Arata, et al, Neurosurgical robotic system for brain tumor removal, Int J CARS, (2011) 6, 375-385

[12] Marcu, C., et al., Motion Algorithms for an Industrial Robot Graphical Simulator, IEEE International Conference on Automation, Quality and Testing, Robotics, (2010), Vol. 1, pp. 14 , DOI: 10.1109/AQTR.2010.5520864

[13] C. Vaida, B. Gherman, D. Pisla, N. Plitea, A spherical robotic arm for instruments positioning in minimally invasive medical applications, The 2nd IFToMM Asian Conference on Mechanism and Machine Science, (2012), Nov. 7 -10, Tokyo, Japan

[14] Figliolini G., Rea P., Grande S. Kinematic synthesis of rotary machines generated by regular curve polygons. ASME international design engineering technical conferences and CIE conference, August 12-15, Chicago, (U.S.A). Paper DETC2012-71192, 2012.

[15] Ottaviano, E., Vorotnikov, S., Ceccarelli, M., Design improvements and control of a hybrid walking robot, (2011), Robotics and Autonomous Systems, vol. 59(2), pp. 128-141 